\title{
The plasma filling factor of coronal bright points ${ }^{\star}$ Coronal bright points
}

\author{
K. P. Dere \\ Department of Computational and Data Sciences, George Mason University, 4400 University Dr., Fairfax VA, 22030, USA
}

e-mail: kdere@gmu .edu

Received 17 April 2008 / Accepted 18 September 2008

ABSTRACT

\begin{abstract}
Aims. We determine the volumetric plasma filling factor of coronal bright points. Methods. Rastered spectra of the regions of the quiet Sun were recorded by the Extreme ultraviolet Imaging Spectrometer (EIS) during operations with the Hinode satellite. Calibrated intensities of Fe XII lines were obtained and images of the quiet corona were constructed. From the imaged spectra, the emission measures and geometrical widths of coronal bright points were obtained. Electron densities were determined from density-sensitive line ratios. A comparison of the emission measure and bright point widths with the electron densities yielded the plasma-filling factor.

Results. The median electron density of coronal bright points is $4 \times 10^{9} \mathrm{~cm}^{-3}$ at a temperature of $1.6 \times 10^{6} \mathrm{~K}$. The volumetric plasmafilling factor of coronal bright points was found to vary from $4 \times 10^{-5}$ to 0.2 with a median value of 0.015 .

Conclusions. The current set of EIS coronal bright point observations indicates that the median value of their plasma filling-factor is 0.015. This can be interpreted as evidence of a considerable subresolution structure in coronal bright points or as the result of a single completely-filled plasma loop with a width on the order of 1-2 arcsec that has not been spatially resolved in these measurements.
\end{abstract}

Key words. Sun: corona

\section{Introduction}

Coronal brights points provide a simplified environment for studying coronal heating. Since bright points were first discovered in EUV (Tousey \& Koomen 1971) and X-ray (Vaiana et al. 1973) observations of the corona, it has been shown that they consist of one or a few small plasma loops with individual lifetimes of 6 min (Sheeley \& Golub 1979), although their typical aggregate lifetime is about $8 \mathrm{~h}$ (Golub et al. 1974). They generally span small bipoles observed in photospheric magnetograms (Krieger et al. 1971; Tousey et al. 1973), which are more often the result of the collision of magnetic flux elements than of flux emergence (Webb et al. 1993). Parker (1988) has suggested that coronal heating occurs through the dissipation of magnetic discontinuities on very fine scales. The goal of this paper is to examine the Extreme ultraviolet Imaging Spectrometer (EIS) observations of coronal bright points and to use the available quantitative diagnostics to determine the densities, temperatures, geometries and plasma filling factors of these structures. In particular, the value of the plasma filling factor should be a direct test of Parker's prediction of nanoflare heating.

\section{Instrumentation, observations and data reduction}

EIS consists of an off-axis, parabolic-mirror telescope which focuses an image of the Sun onto the slit of a normal-incidence,

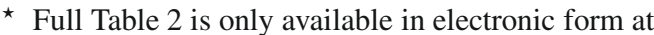
http://www . aanda.org toroidal-grating spectrometer. Two sets of molybdenum-silicon multilayer coatings have been applied to two halves of the mirror and grating to enhance their reflectivity in two extreme ultraviolet bands, nominally 170-210 and 250-290 , the short wavelength $(S W)$ and long wavelength (LW) bands, respectively. Two aluminum filters are used to reject visible light. Both wavelength bands are detected by their individual CCD detector. Each CCD pixel views an angle of 0.9984 arcsec along the slit and $22.3 \mathrm{~m} \AA$, the equivalent of $26 \mathrm{~km} \mathrm{~s}^{-1}$ in the $S W$ band and $36 \mathrm{~km} \mathrm{~s}^{-1}$ in the $L W$ band, along the dispersion. The observations discussed here were all made with a 1 arcsec wide slit. The pointing stability of EIS, as a result of spacecraft pointing variations, is estimated to be 3-5 arcsec. The EIS instrument has been described in greater detail by Culhane et al. (2007) and Hinode has been described by Kosugi et al. (2007).

The observations that will be discussed were made on 2007 June 15-18. They consist of EIS slit rasters over 55 arcsec. wide regions of the quiet Sun with exposure times of 60s. The CCD data from the central 512 pixels were transmitted for 18 spectral windows. Several sets of observations were obtained. On June 15,16 , a set of 5 consecutive, contiguous 55 arcsec rasters were performed to cover a region about $512 \times 275$ arcsec, north-south by east-west. On June 16, 4 series of 3 consecutive, contiguous 55 arcsec rasters were performed to cover a region about $512 \times 165$ arcsec. Finally, on June 17,18 a time series of 20 rasters was performed to cover a region about $512 \times 55$ arcsec over a period of about $37 \mathrm{~h}$ with a somewhat irregular cadence. The field of view of all the observations followed the same region on the Sun as controlled by the Hinode satellite pointing that followed the differential rotation of the Sun. The observations 
were obtained during the Hinode eclipse season so that it was not always possible to observe an unocculted Sun for a full $55 \mathrm{~min}$. The data were further degraded by observations made during passage through the South Atlantic Anomaly and the polar belts as well as the synoptic off-pointings to disk center made 4 times a day.

Data reduction started with the use of the "Level-0" files produced by the standard data production pipeline. A dark, zerosecond exposure obtained on June 1 was subtracted from the Level-0 data by matching their cumulative histograms at the $20 \%$ level. Hot pixels were identified from files in the SSW SolarSoft distribution. Further, "warm" pixels were identified from $100 \mathrm{~s}$ dark exposures obtained on June 19. A warm pixel is defined as one that has a reproducible dark current that can add up to 10 or more DN for a given exposure time.

The slit image is straight but tilted over the extent of the CCD that was read out. The wavelength scale was corrected for the slit tilt. A wavelength scale has been adopted under the assumption that coronal radial velocities are zero when averaged along the slit. An additional complication to the determination of a velocity scale is the variation of the wavelength scale on a time scale that is roughly equal to the orbital period. Consequently, the wavelength scale must be corrected for each exposure. Here, the Fe XII $195 \AA$ line was used for the velocity standard.

Finally, the spectral line profiles were fit with Gaussian profiles by means of a Levenberg-Marquardt algorithm. For the Fe XIII $\lambda 203.8$ lines a two Gaussian fit was used. For all other line profiles discussed here, a single Gaussian fit was used. Young et al. (2008) have discussed the shape of these line profiles and the presence of blends in some detail. They suggest that for densities below $10^{10} \mathrm{~cm}^{-3}$, the case seen here, that the blends to the Fe XII $\lambda 195$ line are not important and that a single Gaussian fit can be used. For the Fe XIII $\lambda 203.8$ lines they used a three-Gaussian fit but found it necessary to constrain some of the parameters of the fit. Here, a two-Gaussian fit was used. An examination of the two-Gaussian fits with respect to the observations indicates that they reproduce the line profile at a level commensurate with the data quality which is limited by the low count rates in the quiet Sun. The statistical weights used in the fitting were derived from the quadrature sum of the photon noise and the CCD read noise. Pixels that were identified as hot or warm pixels were given a weight of zero. The preflight absolute intensity calibration was applied to the data.

The area covered by the central EIS raster is outlined in the Extreme ultraviolet Imaging Telescope (EIT) image shown in Fig. 1. The EIT images were co-aligned to the EIS spectra by means of the EIS He II $\lambda 256$ image and the EIT He II $\lambda 304$ image. The accuracy of the co-alignment is on the order of 2.6 arcsec, the size of an EIT pixel.

\section{Electron densities and filling factors in coronal bright points}

The 5 part raster obtained on June 15,16 is shown in Fig. 2. The raster sequence was started at 1639 UT and finished on June 16 at 0256 UT. The intensities shown in Fig. 2 were logarithmically scaled as were the electron densities. The electron densities have been determined using the scattering calculation of Storey et al. (2005) included in the CHIANTI database (Dere et al. 1997; Landi et al. 2006) for the Fe XII $\lambda 186 / \lambda 195$ ratio. Both lines consist of blends of two Fe XII lines. A previous analysis of EIS spectra (Dere et al. 2007) showed that the rms error in the determination of densities in the quiet Sun was a factor

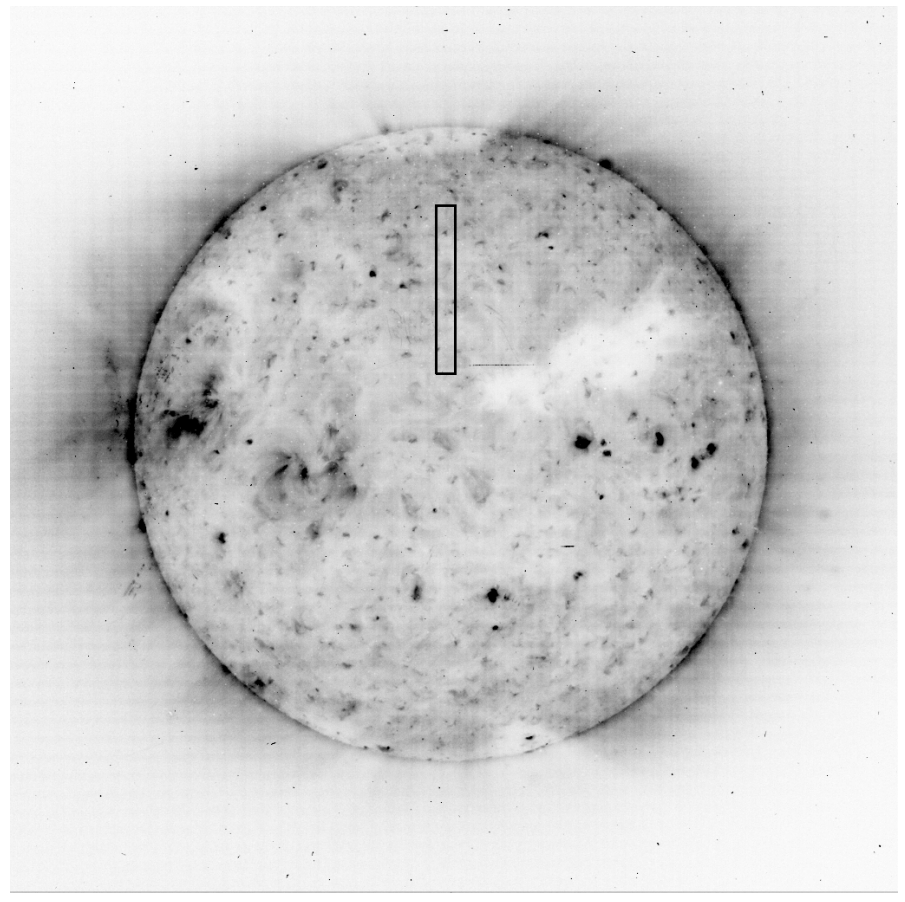

Fig. 1. EIT image in Fe XII $\lambda 195$ with an outline of the area of the central EIS raster.

of 1.6. There is considerably more noise in the density image than in the intensity image, reflecting the lower signal-to-noise ratio of the weaker $186 \AA$ line. Further, Dere et al. (2007) and Young et al. (2008) found that densities derived from the Fe XII line ratio were factors of 1.6 and 1.9, greater than those derived from Fe XIII line ratios, respectively. An analysis of the current data sets indicates that the densities derived from the Fe XII ratios are also about a factor of 1.9 higher than those derived from Fe XIII. The dotted vertical stripes on the left sides of the second and third raster show the effects of satellite night and the bright vertical stripe in the fifth raster is the result of the satellite offpointing to disk center. A previous study of the quiet sun with EIS spectra (Dere et al. 2007) showed a general correlation between coronal bright points and high electron density. In Fig. 2, a number of coronal bright points have been labeled for later discussion. Compared with the great majority of coronal bright points in the figure, that labeled A is considerably larger but will be included in the analysis. The bright points are typically discernible as small loop-like features that have high densities as seen in the density image.

During the period June 17, 18, a time series of EIS rasters was obtained at a single pointing following solar differential rotation. The time series of Fe XII intensity and density images are shown in Fig. 3. In this figure, coronal bright points have also been labeled. The numbers 0 through 18 refer to the series of rasters whose observations times are shown in Table 1 . The letters refer to bright points that are seen in one or more rasters. In some rasters, the bright points are discernible as a collection of 2 or more loops and each loop has been further labeled with an $a$, $b$, or $c$.

The plasma filling factor for each bright point observation was derived by measuring the peak intensity in Fe XII $\lambda 195$, a typical electron density near the center of the bright point, and the bright point width. Because the bright points are near the resolution of EIS, there are generally not a sufficient number of data to determine a true average value of the electron density. 

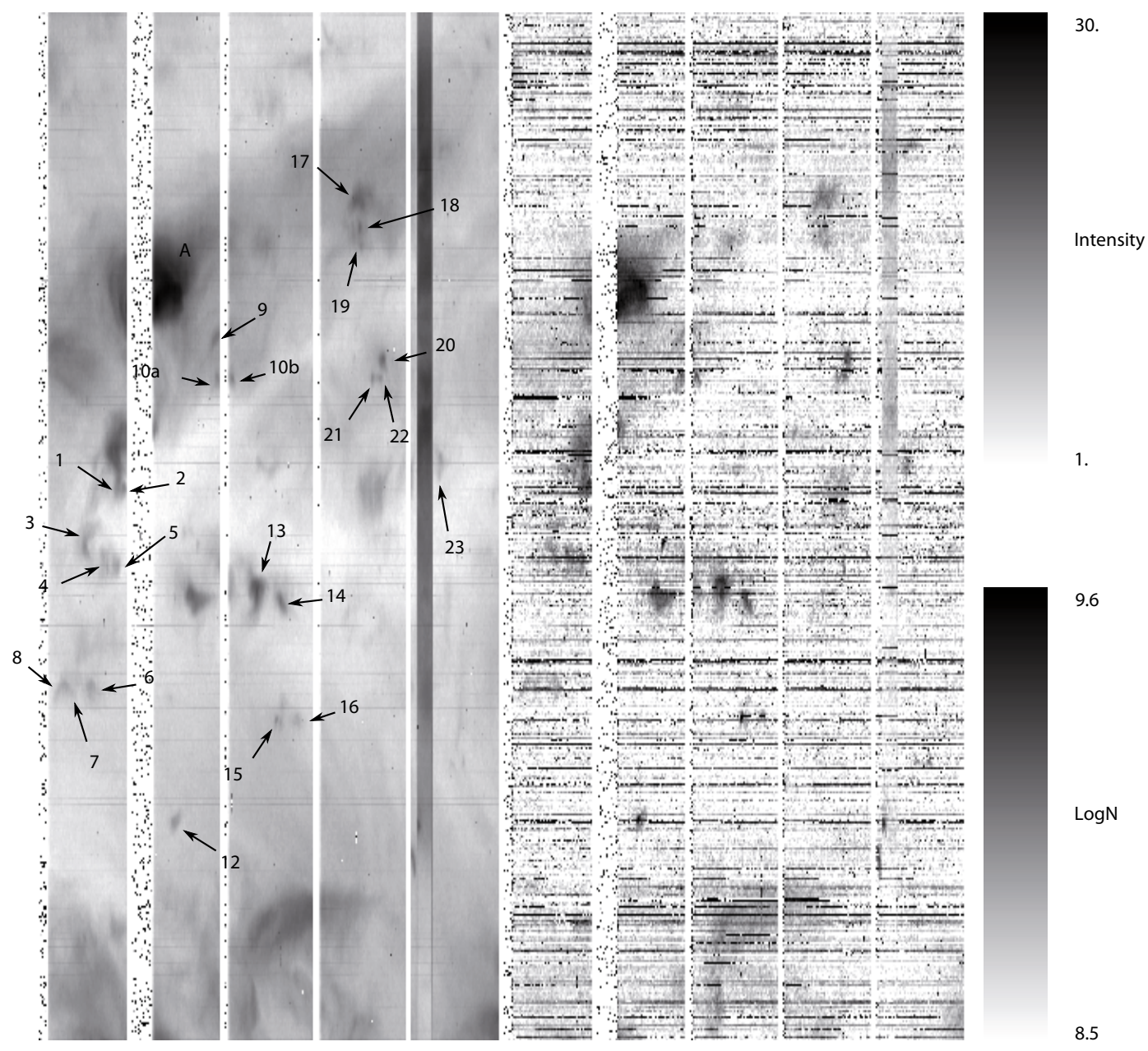

Fig. 2. Left, Fe XII $\lambda 195$ intensity images, and, right, electron density images derived from the Fe XII density-sensitive line-ratio pair.

Table 1. June 17,18 observation times.

\begin{tabular}{llll}
\hline \hline Raster & Day & Start time (UT) & End time (UT) \\
\hline 0 & 17 & 0247 & 0345 \\
1 & 17 & 0423 & 0521 \\
2 & 17 & 0623 & 0721 \\
3 & 17 & 0743 & 0841 \\
4 & 17 & 0920 & 1018 \\
5 & 17 & 1059 & 1157 \\
6 & 17 & 1236 & 1334 \\
7 & 17 & 1416 & 1514 \\
8 & 17 & 1615 & 1713 \\
9 & 17 & 1752 & 1850 \\
10 & 17 & 2242 & 2340 \\
11 & 18 & 0019 & 0117 \\
12 & 18 & 0146 & 0244 \\
13 & 18 & 0322 & 0420 \\
14 & 18 & 0501 & 0559 \\
15 & 18 & 0640 & 0738 \\
16 & 18 & 1136 & 1234 \\
17 & 18 & 1317 & 1415 \\
18 & 18 & 1458 & 1556 \\
\hline
\end{tabular}

The width is the full width at half maximum of the intensity profile perpendicular to the length of the loop and has an estimated accuracy of about 30\%. These data are listed in Table 2. As the complete table is only available electronically, only two sample entries are shown here. The median value of the bright
Table 2. Coronal bright point intensities, densities, widths and filling factors. The full table is only available in electronic form.

\begin{tabular}{llllll}
\hline \hline Label & Raster & Intensity $^{a}$ & Log density $^{b}$ & Width $^{c}$ & Fill factor \\
\hline 1 & $\mathrm{Z}$ & $1.65 \mathrm{e}+02$ & 9.42 & 5.0 & $6.7 \mathrm{e}-02$ \\
$\mathrm{~B}$ & 2 & $1.51 \mathrm{e}+02$ & 10.32 & 4.6 & $1.0 \mathrm{e}-03$ \\
\hline
\end{tabular}

${ }^{a} \operatorname{erg~} \mathrm{cm}^{-2} \mathrm{~s}^{-1} \mathrm{sr}^{-1} ;{ }^{b} \mathrm{~cm}^{-3} ;{ }^{c} \operatorname{arcsec}$.

point widths is 5 arcsec. The raster listing refers to the number in Table 1 with the raster $z$ referring to the set of 5 rasters obtained on June 15,16 . In a large number of bright points, the width is apparently that of a single coronal loop. loop is

The observed intensity of a spectral line emitted by a coronal

$I=\int G(T) N_{\mathrm{e}} N_{\mathrm{H}} \mathrm{d} l$

where $N_{\mathrm{e}}$ is the electron density, $N_{\mathrm{H}}$ is the hydrogen density and $G(T)$ is the contribution function, calculated with the CHIANTI database. To apply this to a resolved structure such as a coronal bright point, it is assumed that the feature is isothermal and the $G(T)$ function is evaluated at its peak temperature $T_{\max }$. For a temperature of $1.6 \times 10^{6} \mathrm{~K}$, appropriate for Fe XII, the hydrogen density $N_{\mathrm{H}}=0.86 N_{\mathrm{e}}$. The path length of the integration is the loop width $w$ under the assumption that the bright point is a cylindrical loop. If the loop is not completely filled with emitting 

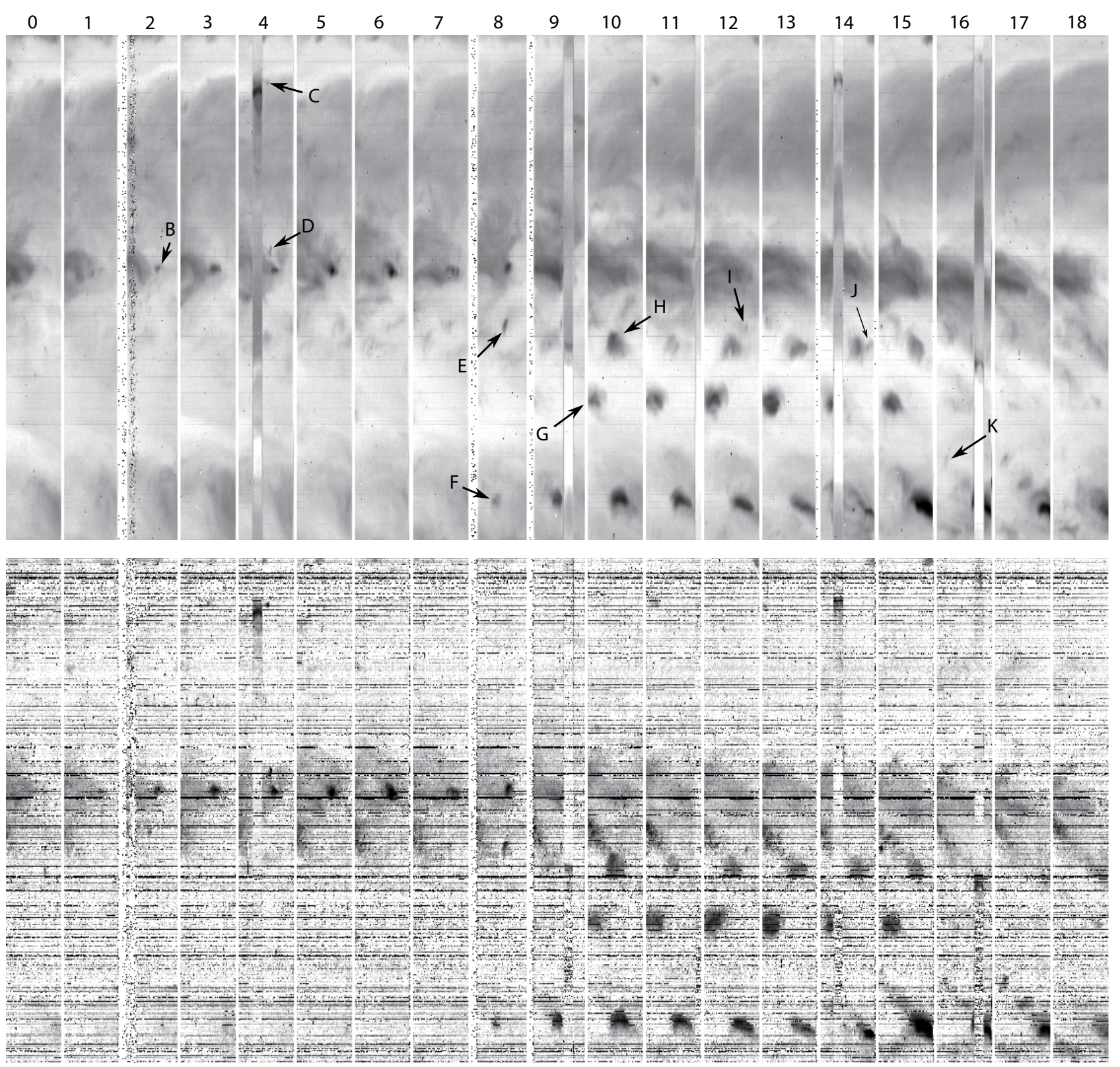

Fig. 3. Top, a time series of Fe XII $\lambda 195$ intensity images, and, bottom, a time series of electron density images derived from the Fe XII densitysensitive line-ratio pair. The numeric labels refer to the raster labels in Table 1.

plasma, then it necessary to introduce a volumetric filling factor $\mathrm{f}$ to account for this. Under these assumptions

$I=0.86 G\left(T_{\max }\right) N_{\mathrm{e}}^{2} f w$.

In Table 2, the observables needed to derive the filling factor $f$ are provided and the values of the resulting filling factor listed. A background intensity value has been subtracted to arrive at the line intensities listed in the table. The background value is an typical value of the line intensity in the neighborhood of the bright point. Separate background values were applied to both the Fe XII $186 \AA$ and $195 \AA$ lines. This background is interpreted as the result of multiple large scale structures overlying the bright points. A histogram of electron densities is shown in Fig. 4. The median electron density is $4 \times 10^{9} \mathrm{~cm}^{-3}$. The distribution of filling factors is shown in Fig. 5 and the median value of the filling factor is 0.015 . If the electron densities are actually about a factor of 2 lower, as suggested by the Fe XIII line ratios, then the median fill factor would be 0.06 . Somewhat lower filling factors were found in the transition region using a similar approach (Dere et al. 1987).

The distribution of filling factors shown in Fig. 4 suggests a Gaussian-shaped distribution. However, it is clear that this is the result of a real distribution of filling factors and not the result of accumulated errors in the measurements. For example, bright point $\mathrm{F}$ has electron densities that generally range in value between $10^{9.6}$ to $10^{10.1}$ while bright point $\mathrm{H}$ has density values that range between $10^{9.3}$ and $10^{9.6}$, consistently lower than those in $\mathrm{F}$. Generally, individual bright points show fairly consistent variations in density and width with time. The largest changes in the filling factor come about when the bright point can be seen to break up into multiple bright points.

\section{Discussion}

From the analysis of the EIS spectra of coronal bright points, filling factors significantly less than unity are found. Perhaps the 


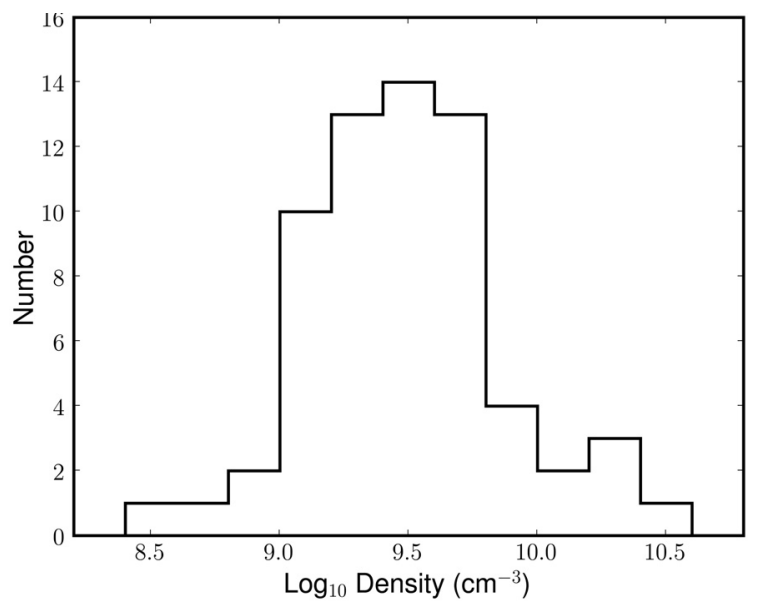

Fig. 4. Histogram of coronal bright point electron densities.

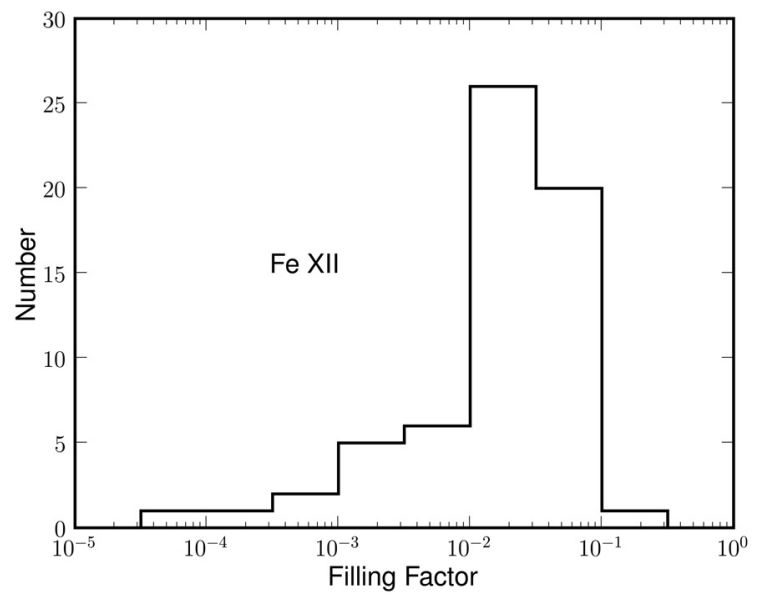

Fig. 5. Histogram of coronal bright point filling factors.

most questionable assumption in this analysis is that the Fe XII emission actually occurs near the peak of the Fe XII contribution function. The coronal bright points observed here were also apparent in images obtained with the X-ray telescope (XRT) on Hinode. The X-ray emission is likely to be produced at temperatures well above $1.6 \times 10^{6} \mathrm{~K}$. In order to examine this question, the isothermal emission measures for a variety of lines were determined for bright point $\mathrm{F}$ observed in raster 12 . These are shown in Fig. 6. It is evident that, for this brightpoint, $1.6 \times 10^{6} \mathrm{~K}$ is near the peak of the emission-measure distribution.

Ugarte-Urra (2004) and Ugarte-Urra et al. (2005) have used the Coronal Diagnostic Spectrometer (CDS) on SOHO to measure electron densities in coronal bright points. For Fe XII they find densities ranging from $10^{9.2}$ to $10^{9.9} \mathrm{~cm}^{-3}$ when background intensities are subtracted. These values are in general agreement with our current results. Further, Ugarte-Urra (2004) measured the widths of coronal bright points in the $171 \AA$ TRACE images and found an average $F W H M$ of 3 arcsec and length of 8 arcsec. These images are formed predominantly by emission in Fe IX and $\mathrm{Fe} \mathrm{X}$ at $1 \times 10^{6} \mathrm{~K}$.

The low plasma filling factors found here indicate that there is significant fine structure below the resolution of these observations. One way to interpret this result is to assume that all of the plasma is located in a single, completely-filled loop with the same length as the observed loop but with a smaller diameter. Under this assumption, the FWHM of this single loop is the observed $F W H M$ multiplied by the square-root of the volumetric

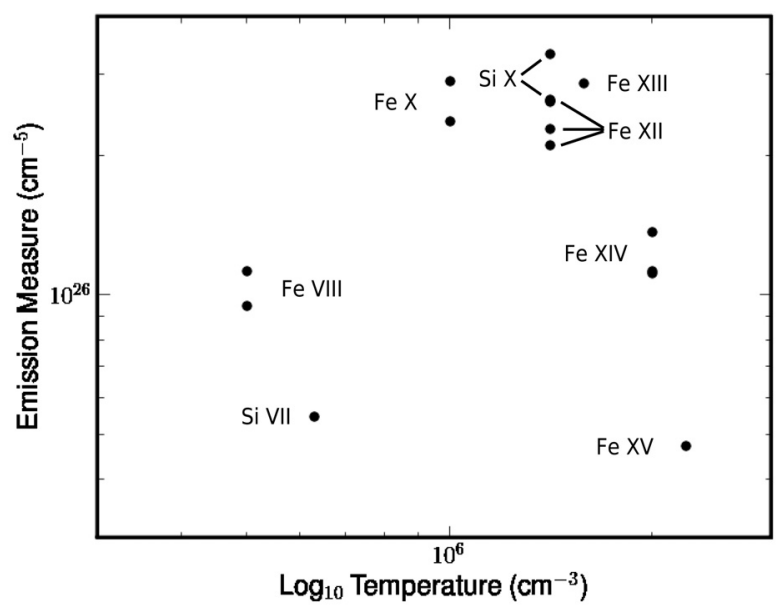

Fig. 6. Isothermal emission measures for a bright point.

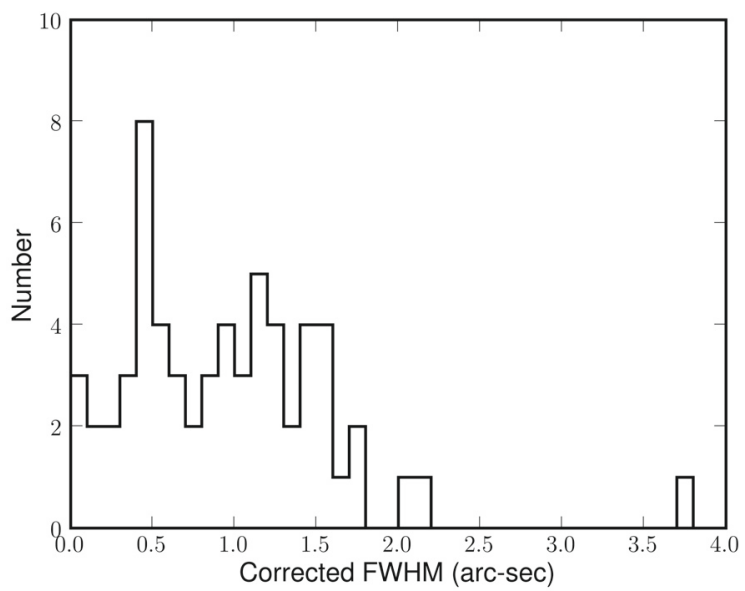

Fig. 7. Histogram of the subresolution $F W H M$ of the observed bright points.

filling factor. Basically, the volume of this single subresolution loop is taken to be the observed length times the reduced area. For all of the entries in Table 2, the subresolution FWHM has been calculated and the distribution of these values is shown in Fig. 7. The median value of the subresolution FWHM is 0.9 arcsec. Again, if the electron densities are actually about a factor of 2 lower, as suggested by the Fe XIII line ratios, then the median subresolution $F W H M$ would be 1.8 arcsec. TRACE observations (Ugarte-Urra 2004) reveal bright points widths of 3 arcsec. that are below the 5 arcsec. widths measured with EIS but somewhat larger than those predicted for a single subresolution, filled loop. However, the TRACE measurements also refer to emission at $1 \times 10^{6} \mathrm{~K}$ which often shows finer structures than observed at $1.6 \times 10^{6} \mathrm{~K}$ in Fe XII. Consequently, corona bright points may consist of many fine-scale structures below their apparent sizes determined from recent measurements, but they may also be interpreted as single completely filled loops with crosssectional dimensions somewhat below the spatial resolution of current observations.

These results suggest that further investigations of coronal bright point would be worthwhile. TRACE observations in the Fe XII $195 \AA$ band were made at the same time as the EIS observations. Measurements of the widths of the same loops listed in Table 2 would be useful. It is possible that the bright point dimensions determined from the EIS observations are broadened by variations in the spacecraft pointing. The distribution of 
emission measures shown in Fig. 6 is quite narrow. Aschwanden (2002) suggests that the loops observed in the different TRACE bands are isothermal. One question that should be examined is whether the observed line intensities could be reproduced by a single isothermal loop. In this context, it would also be useful to address any temperature structure in the direction transverse to the main axis of the observed loops.

\section{Conclusions}

EIS spectra of coronal bright points have been examined. The intensities and geometrical widths have been measured. Emission measures have been determined from line intensities and electron densities determined from density-sensitive line ratios. A comparison of the electron densities derived from the loop emission measures and widths with the densities derived from the density-sensitive line ratios indicates that the volumetric plasma filling factor of the coronal bright points ranges from about $4 \times 10^{-5}$ to 0.2 with a median value of 0.015 . If the observed bright points are interpreted as single, completely filled loops, their median widths would be on the order of 1 to 2 arcsec, somewhat below the EIS spatial resolution. Consequently, the existence of subresolution fine structure in coronal bright points can not be ruled out but neither can it be confirmed. The continued analysis of EIS, TRACE and other observations will be valuable.

Acknowledgements. Hinode is a Japanese mission developed and launched by ISAS/JAXA, with NAOJ as domestic partner and NASA and STFC (UK) as international partners. It is operated by these agencies in co-operation with ESA and NSC (Norway). We are grateful for the use of EIT data obtained on the SOHO spacecraft. SOHO is a project of international cooperation between ESA and NASA. This research has made use of NASA's Astrophysics Data System.

\section{References}

Aschwanden, M. J. 2002, ApJ, 580, L79

Culhane, J. L., Harra, L. K., James, A. M., et al. 2007, Sol. Phys., 243, 19

Dere, K. P., Bartoe, J.-D. F., Brueckner, G. E., Cook, J. W., \& Socker, D. G. 1987, Sol. Phys., 114, 223

Dere, K. P., Landi, E., Mason, H. E., Monsignori Fossi, B. C., \& Young, P. R. 1997, A\&AS, 125, 149

Dere, K. P., Doschek, G. A., Mariska, J. T., et al. 2007, PASJ, 59, 721

Golub, L., Krieger, A. S., Silk, J. K., Timothy, A. F., \& Vaiana, G. S. 1974, ApJ, 189, L93

Kosugi, T., Matsuzaki, K., Sakao, T., et al. 2007, Sol. Phys., 243, 3

Krieger, A. S., Vaiana, G. S., \& van Speybroeck, L. P. 1971, in Solar Magnetic Fields, ed. R. Howard, IAU Symp., 43, 397

Landi, E., Del Zanna, G., Young, P. R., et al. 2006, ApJS, 162, 261

Parker, E. N. 1988, ApJ, 330, 474

Sheeley, Jr., N. R., \& Golub, L. 1979, Sol. Phys., 63, 119

Storey, P. J., Del Zanna, G., Mason, H. E., \& Zeippen, C. J. 2005, A\&A, 433, 717

Tousey, R., \& Koomen, M. J. 1971, Sol. Phys., 21, 401

Tousey, R., Bartoe, J. D. F., Bohlin, J. D., et al. 1973, Sol. Phys., 33, 265

Ugarte-Urra, I. 2004, Ph.D. Thesis, AA. Armagh Observatory, College Hill, Armagh BT61 9DG, N. Ireland, UK

Ugarte-Urra, I., Doyle, J. G., \& Del Zanna, G. 2005, A\&A, 435, 1169

Vaiana, G. S., Davis, J. M., Giacconi, R., et al. 1973, ApJ, 185, L47

Webb, D. F., Martin, S. F., Moses, D., \& Harvey, J. W. 1993, Sol. Phys., 144, 15

Young, P. R., Watanabe, T., Hara, H., \& Mariska, J. T. 2008, ArXiv e-prints, 805 
K. P. Dere: The plasma filling factor of coronal bright points, Online Material $p 1$

Table 2. Coronal bright point intensities, densities, widths and filling factors.

\begin{tabular}{|c|c|c|c|c|c|}
\hline Label & Raster & Intensity $^{a}$ & Log density ${ }^{b}$ & Width $^{c}$ & Fill factor \\
\hline 1 & $\mathrm{Z}$ & $1.65 e+02$ & 9.42 & 5.0 & $6.7 \mathrm{e}-02$ \\
\hline 2 & $\mathrm{z}$ & $1.21 \mathrm{e}+02$ & 9.84 & 3.4 & $1.0 \mathrm{e}-02$ \\
\hline 3 & $\mathrm{z}$ & $8.97 e+01$ & 9.34 & 4.4 & $6.0 \mathrm{e}-02$ \\
\hline 4 & $\mathrm{z}$ & $4.66 e+01$ & 9.48 & 5.4 & $1.3 e-02$ \\
\hline 5 & $\mathrm{z}$ & $5.20 \mathrm{e}+01$ & 9.25 & 6.0 & $3.8 \mathrm{e}-02$ \\
\hline 6 & $\mathrm{z}$ & $5.81 \mathrm{e}+01$ & 9.35 & 6.0 & $2.7 \mathrm{e}-02$ \\
\hline 7 & $\mathrm{z}$ & $4.99 e+01$ & 9.30 & 4.0 & $4.4 \mathrm{e}-02$ \\
\hline 9 & $\mathrm{z}$ & $8.25 e+01$ & 9.35 & 3.9 & $5.9 \mathrm{e}-02$ \\
\hline $10 \mathrm{a}$ & $\mathrm{z}$ & $5.63 e+01$ & 10.35 & 2.4 & 6.6e-04 \\
\hline $10 \mathrm{~b}$ & $\mathrm{z}$ & $1.00 \mathrm{e}+02$ & 9.47 & 2.8 & $5.8 \mathrm{e}-02$ \\
\hline 11 & $\mathrm{z}$ & $1.99 \mathrm{e}+02$ & 9.46 & 4.8 & 7.0e- -02 \\
\hline 12 & $\mathrm{z}$ & $6.10 \mathrm{e}+01$ & 10.44 & 5.4 & $2.1 \mathrm{e}-04$ \\
\hline 13 & $\mathrm{z}$ & $2.80 \mathrm{e}+02$ & 9.71 & 8.2 & $1.8 \mathrm{e}-02$ \\
\hline 14 & $\mathrm{z}$ & $1.65 e+02$ & 9.48 & 3.6 & 7.0e- 02 \\
\hline 15 & $\mathrm{z}$ & $8.39 \mathrm{e}+01$ & 9.21 & 2.4 & $1.9 \mathrm{e}-01$ \\
\hline 17 & $\mathrm{z}$ & $1.36 e+02$ & 9.5 & 4.8 & 4.0e- -02 \\
\hline 18 & $\mathrm{z}$ & $1.33 e+02$ & 9.63 & 2.8 & $3.6 \mathrm{e}-02$ \\
\hline 19 & $\mathrm{z}$ & $1.00 \mathrm{e}+02$ & 9.4 & 3.1 & $7.2 \mathrm{e}-02$ \\
\hline 20 & $\mathrm{z}$ & $1.79 \mathrm{e}+02$ & 9.74 & 4.0 & $2.1 \mathrm{e}-02$ \\
\hline 21 & $\mathrm{z}$ & $8.25 e+01$ & 9.95 & 3.0 & $4.8 \mathrm{e}-03$ \\
\hline 22 & $\mathrm{z}$ & $8.61 e+01$ & 9.68 & 2.4 & $2.2 \mathrm{e}-02$ \\
\hline 23 & $\mathrm{z}$ & $7.53 e+01$ & 9.7 & 4.0 & $1.0 \mathrm{e}-02$ \\
\hline A & $\mathrm{z}$ & $8.25 e+02$ & 9.68 & 28.0 & $1.8 \mathrm{e}-02$ \\
\hline B & 2 & $1.51 \mathrm{e}+02$ & 10.32 & 4.6 & $1.0 \mathrm{e}-03$ \\
\hline B & 3 & $2.30 \mathrm{e}+02$ & 10.13 & 10.0 & $1.8 \mathrm{e}-03$ \\
\hline B & 4 & $2.65 e+02$ & 10.21 & 7.0 & $2.0 \mathrm{e}-03$ \\
\hline B & 5 & $7.10 \mathrm{e}+02$ & 10.2 & 4.3 & $9.2 \mathrm{e}-03$ \\
\hline B & 6 & $7.39 \mathrm{e}+02$ & 10.17 & 4.0 & $1.2 \mathrm{e}-02$ \\
\hline B & $7 \mathrm{a}$ & $2.08 \mathrm{e}+02$ & 9.72 & 4.0 & $2.6 \mathrm{e}-02$ \\
\hline B & $7 b$ & $2.30 \mathrm{e}+02$ & 9.72 & 6.0 & $1.9 \mathrm{e}-02$ \\
\hline B & $7 \mathrm{c}$ & $2.04 e+02$ & 9.72 & 5.0 & $2.1 \mathrm{e}-02$ \\
\hline B & 8 & $3.66 e+02$ & 9.97 & 5.8 & $1.0 \mathrm{e}-02$ \\
\hline $\mathrm{C}$ & 4 & $8.97 e+01$ & 11.2 & 1.4 & $3.6 \mathrm{e}-05$ \\
\hline E & 8 & $1.90 \mathrm{e}+02$ & 9.74 & 6.1 & $1.4 \mathrm{e}-02$ \\
\hline $\mathrm{F}$ & 8 & $1.26 \mathrm{e}+02$ & 9.45 & 6.2 & $3.6 \mathrm{e}-02$ \\
\hline $\mathrm{F}$ & 9 & $2.87 e+02$ & 10.1 & 9.0 & $2.8 \mathrm{e}-03$ \\
\hline $\mathrm{F}$ & $10 \mathrm{a}$ & $5.06 e+02$ & 9.67 & 7.8 & $4.1 \mathrm{e}-02$ \\
\hline $\mathrm{F}$ & $10 \mathrm{~b}$ & $4.09 \mathrm{e}+02$ & 9.67 & 6.6 & $4.0 \mathrm{e}-02$ \\
\hline $\mathrm{F}$ & 11 & $4.41 e+02$ & 9.6 & 11.2 & $3.5 e-02$ \\
\hline $\mathrm{F}$ & 12 & $4.38 \mathrm{e}+02$ & 9.7 & 9.4 & $2.6 \mathrm{e}-02$ \\
\hline $\mathrm{F}$ & 13 & $3.06 e+02$ & 9.7 & 9.2 & $1.9 \mathrm{e}-02$ \\
\hline $\mathrm{F}$ & 14 & $3.95 e+02$ & 10.5 & 9.0 & $6.1 \mathrm{e}-04$ \\
\hline $\mathrm{F}$ & 15 & $1.40 \mathrm{e}+03$ & 9.97 & 11.0 & $2.0 \mathrm{e}-02$ \\
\hline $\mathrm{F}$ & 17 & $7.89 \mathrm{e}+02$ & 9.87 & 9.0 & $2.2 \mathrm{e}-02$ \\
\hline G & 10 & $1.74 \mathrm{e}+02$ & 9.65 & 11.0 & $1.1 \mathrm{e}-02$ \\
\hline G & 11 & $2.26 \mathrm{e}+02$ & 9.65 & 8.0 & $2.0 \mathrm{e}-02$ \\
\hline G & 12 & $3.37 e+02$ & 9.6 & 9.8 & $3.0 \mathrm{e}-02$ \\
\hline G & 13 & $3.30 \mathrm{e}+02$ & 9.67 & 11.2 & $1.9 \mathrm{e}-02$ \\
\hline G & 15 & $2.73 e+02$ & 9.65 & 15.4 & $1.2 \mathrm{e}-02$ \\
\hline $\mathrm{H}$ & $10 \mathrm{a}$ & $1.84 \mathrm{e}+02$ & 9.5 & 8.6 & $3.0 \mathrm{e}-02$ \\
\hline $\mathrm{H}$ & $10 \mathrm{~b}$ & $1.97 e+02$ & 9.5 & 8.0 & $3.5 \mathrm{e}-02$ \\
\hline $\mathrm{H}$ & $11 \mathrm{a}$ & $5.31 \mathrm{e}+01$ & 9.6 & 5.0 & $9.4 \mathrm{e}-03$ \\
\hline $\mathrm{H}$ & $11 \mathrm{~b}$ & $5.38 \mathrm{e}+01$ & 9.6 & 6.8 & 7.0e- -03 \\
\hline $\mathrm{H}$ & $12 \mathrm{a}$ & $1.37 \mathrm{e}+02$ & 9.43 & 6.2 & $4.3 e-02$ \\
\hline $\mathrm{H}$ & $12 b$ & $9.11 \mathrm{e}+01$ & 9.43 & 6.0 & $2.9 \mathrm{e}-02$ \\
\hline $\mathrm{H}$ & 13 & $1.36 e+02$ & 9.55 & 18.0 & $8.4 \mathrm{e}-03$ \\
\hline $\mathrm{H}$ & 14 & $1.65 e+02$ & 9.4 & 6.0 & $6.1 \mathrm{e}-02$ \\
\hline $\mathrm{H}$ & $15 \mathrm{a}$ & $1.11 \mathrm{e}+02$ & 9.3 & 5.4 & $7.2 \mathrm{e}-02$ \\
\hline $\mathrm{H}$ & $15 b$ & $1.29 \mathrm{e}+02$ & 9.3 & 10.0 & $4.5 \mathrm{e}-02$ \\
\hline I & 12 & $6.82 \mathrm{e}+01$ & 9.6 & 2.9 & $2.1 \mathrm{e}-02$ \\
\hline $\mathrm{J}$ & 15 & $1.02 \mathrm{e}+02$ & 10.16 & 2.2 & $3.1 \mathrm{e}-03$ \\
\hline K & 17 & $8.25 e+01$ & 9.73 & 4.6 & $8.7 e-03$ \\
\hline
\end{tabular}

${ }^{a} \mathrm{erg} \mathrm{cm}^{-2} \mathrm{~s}^{-1} \mathrm{sr}^{-1},{ }^{b} \mathrm{~cm}^{-3},{ }^{c}$ arc-sec. 